\title{
DATA MINING FOR INTERFERENCE AVOIDANCE IN SMART CITIES IOT NETWORKS
}

\author{
UDC ((004.738.5:004.451.53:004.22):004.738.5.057.4)
}

\section{Valentina Nejković, Nenad Milošević, Filip Jelenković, Zorica Nikolić, Milorad Tošić}

\author{
University of Niš, Faculty of Electronic Engineering, Niš, Republic of Serbia
}

\begin{abstract}
Rapid growth of wireless communications and heavily occupied spectrum lead to an inevitable interference between the heterogenous systems operating in the same frequency band. Having in mind the development of the Internet of Things (IoT) services and networks and widely present WiFi networks on the one hand, and the fact that these two systems occupy the same $2.4 \mathrm{GHz}$ frequency band on the other hand, it is clear that the control of the interference and the spectrum coordination are of the highest importance. The first step in the interference control is to acquire its properties. Since the simulation of a large IoT network is not entirely possible, due to the numerous factors not known in advance, the interference assessment is performed on the SmartSantander, an IoT testbed, located in Santander, Spain. This paper presents a statistical analysis of the sensor data and describes the interference properties and its influence. These results may be used for the spectrum coordination, together with the neural networks and semantic technologies.
\end{abstract}

Key words: Coordination, Internet of things, semantic technologies, WiFi, ZigBee

\section{INTRODUCTION}

Integration of computers into every aspect of human's life is an idea that has been known for almost 40 years [1]. However, the blending of computers, as were known before, has progressed into the blending of various smart devices within a network, such as the network of sensors. This evolution in the area especially erupted in the past 20 years [2], influenced by the development of the wireless communication technologies and the Internet, establishing what we now call the Internet of Things (IoT) [3], [4]. The IoT is realized with a lot of small low-cost devices with computing capabilities, which have various actuators or sensors.

Received January 24, 2018

Corresponding author: Nenad Milošević

University of Niš, Faculty of Electronic Engineering, Aleksandra Medvedeva 14, 18000 Niš, Republic of Serbia

E-mail: nenad.milosevic@elfak.ni.ac.rs 
The communication subsystem makes an important part of any IoT system [5]. There are a lot of different communication environments and application areas. Therefore, a plethora of communication technologies exist. Some of these technologies are developed for the specific IoT applications, such as ZigBee [6], [7] and Bluetooth low energy [8], [9]. These communication schemes are widely used, but there is a problem: they operate in $2.4 \mathrm{GHz}$ frequency band, which is heavily used by IEEE $802.11 \mathrm{WiFi}$ devices.

Apparently, the problem of interference between WiFi and ZigBee is very significant, especially in the uncontrolled environments with a large number of devices. A good example of such an environment is increasingly popular smart cities. A smart city is an urban area that uses different types of electronic data collection sensors to supply information used to manage assets and resources efficiently. This includes data collected from citizens, devices, and assets that are processed and analyzed to monitor and manage traffic and transportation systems, power plants, water supply networks, waste management, law enforcement, information systems, schools, libraries, hospitals, and other community services. The smart city concept integrates information and communication technology (ICT), and various physical devices connected to the network (the Internet of things or IoT) to optimize the efficiency of city operations and services and connect to citizens. The interference analysis in smart cities may be done in three different ways. The first and the most affordable approach is to simulate the entire environment. However, the smart city environment is very complex and cannot be accurately described in a simulation. The other approach is to use live commercial installation, but the experimentation in this environment would interfere with normal operation of the system. Finally, the third methodology is to use some of the massive online testbeds or online laboratories. In this paper, the presented analysis is based on real smart city data collected on the FIESTA-IoT platform [10], particularly on the IoT network in SmartSantander testbed [11]. The paper will analyze data from the ZigBee sensors, indicate problems and suggest a possible way to solve these problems.

The rest of the paper is organized as follows. Section 2 discusses the problem of ZigBee and WiFi coexistence. The SmartSantander testbed is described in Section 3, and the statistical analysis of the sensor readings sequences are given in Section 4. The concluding remarks and future work are given in Section 5.

\section{ZIGBEE WIFI COEXISTENCE}

ZigBee is a standard for the wireless networks that uses low-power and may be implemented on inexpensive hardware. It is intended for the application in wireless sensor networks (WSN), and WSNs are the first industrial IoT application. ZigBee was proposed 20 years ago with several revisions after that. ZigBee is based on the IEEE 802.15.4 standard and operates in the $2.4 \mathrm{GHz}$ frequency band. At physical level ZigBee satisfies the low-power needs of IoT networks. On the other hand, MAC level was unreliable and with high energy consumption [12], [13]. Some improvements in the MAC level are part of the IEEE 802.15.4-2012 revision.

WiFi is based on the IEEE 802.11 standard and its subversions. In contrast to ZigBee, $\mathrm{WiFi}$ was not designed to be used in IoT applications. The main purpose of WiFi is to provide high bandwidth to the devices that are in close proximity to each other. Also, WiFi does not support a high number of devices connected to one access point. WiFi is 
not used in IoT primarily because of its high energy consumption, 10 - 100 times higher than ZigBee or Bluetooth. Recently, it has been enhanced regarding the energy consumption, but it is still not widely used for IoT applications.

Even though WiFi is not interesting as a communication subsystem for IoT applications, it is very significant for IoT since it causes interference to ZigBee devices. Namely, WiFi is designed to operate in both 2.4 and $5 \mathrm{GHz}$ frequency bands, with the $2.4 \mathrm{GHz}$ band being heavily used today. Since ZigBee also uses the $2.4 \mathrm{GHz}$ band, mutual interference is inevitable. To understand the potential for problems, a review of the RF spectrums and available channels for WiFi (802.11b/g) and ZigBee (802.15.4) is shown in Fig. 1 [14].

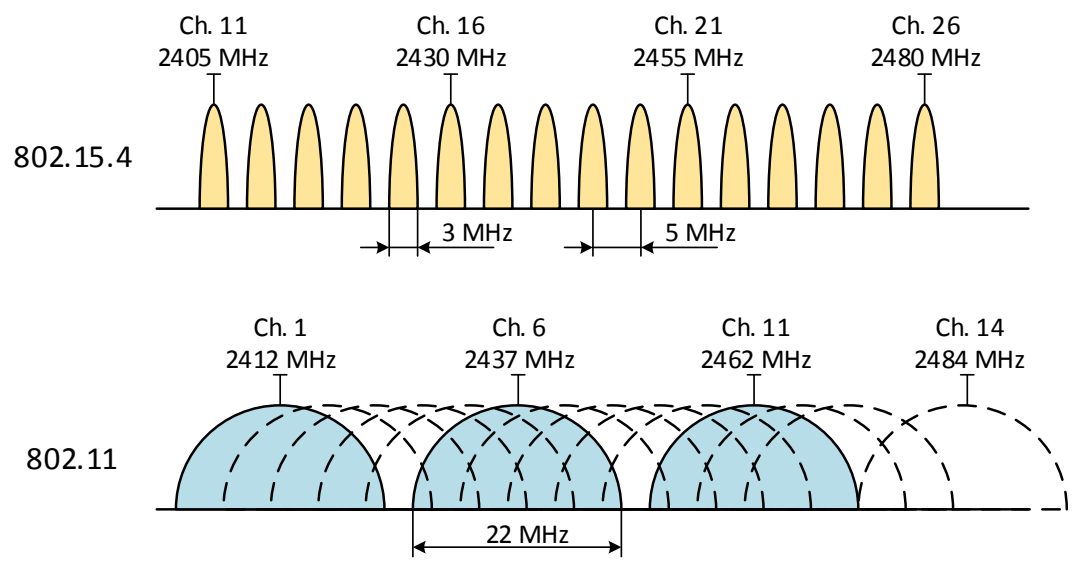

Fig. 1 Comparison of IEEE802.15.4 and IEEE802.11 spectrum occupancy

It is well known that ZigBee communication links experience a large packet loss in case of $\mathrm{WiFi}$ interference. The main reason for this packet loss is the fact that WiFi has much higher transmission power [15]-[17]. The interference may be reduced or completely avoided in space, time or frequency domain. Space domain interference suppression is not very appropriate for ZigBee devices because of a large number of devices in a large area. However, time domain interference avoidance may be used. Although WiFi has carrier-sense multiple access with collision avoidance (CSMA/CA) technology for the avoidance of other active users by sensing the presence of the carrier, WiFi is not able to detect ZigBee [15] because of the ZigBee's low transmit power. Paper [18] proposed the use a separate transmitter, much stronger than ZigBee, that is used to stop WiFi transmission. The drawback of this approach is the lower frequency usage efficiency. Since there are gaps in WiFi transmission, ZigBee may use these gaps for its own transmission [16]. Due to ZigBee devices synchronization problems, the time domain interference avoidance may be used only for the networks of the small number of ZigBee devices. The interference avoidance in the frequency domain is also attractive. The most of the research is focused on ZigBee devices and its channel adjustment [19]-[23], but recently [24] proposed that both ZigBee and WiFi change operating frequency. 


\section{SMARTSANTANDER}

The SmartSantander testbed [11] is located in Santander, Spain. It is an experimental testbed, or open online laboratory, for the research and experimentation in the area of large-scale architectures, in the context of a smart city IoT environment.

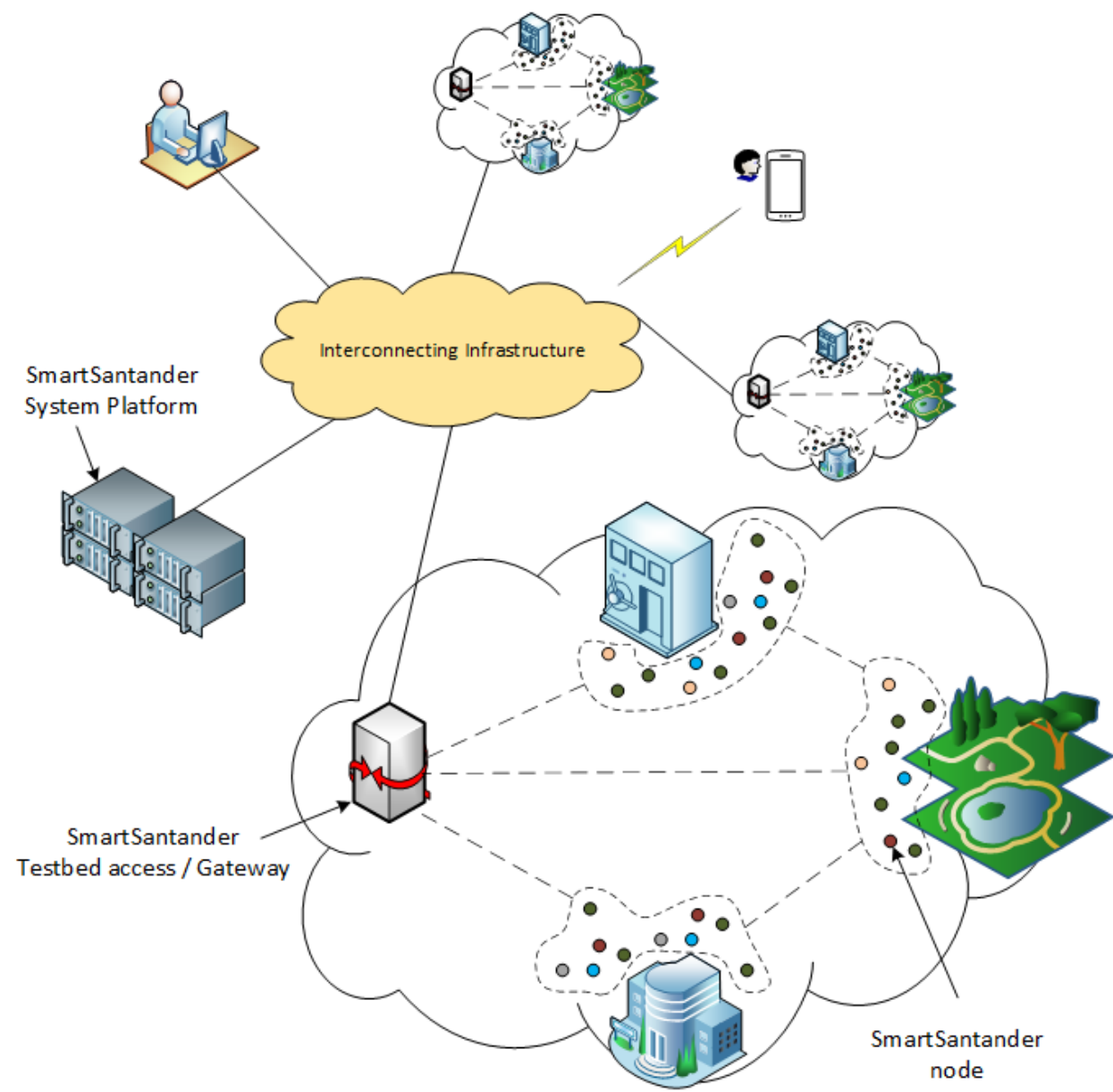

Fig. 2 SmartSantander architecture

The SmartSantander testbed consists of around 3,000 IoT devices that use IEEE 802.15.4 communication protocol. Besides there are more than 2,000 joint Radio Frequency Identification (RFID) tags/Quick Response (QR) code labels and more than 2,000 points of interest at massive gathering places, such as shopping centers, restaurants, cultural events, etc. The architecture of SmartSantander, shown in detail in Fig. 2, consists of three layers. The first layer is comprised of the IoT nodes. Each of them has a sensor for some parameter, such as temperature, carbon-monoxide, moisture, light, car presence, 
etc. The summary of the deployed IoT nodes is shown in Table 1 [11]. The second layer is made of repeaters. The repeaters are placed high above the ground in street lights,

Table 1 SmartSantander IoT nodes [11]

\begin{tabular}{|c|c|c|c|}
\hline Domain & Asset (physical phenomena, etc.) & Resource Type & Deployed devices \\
\hline $\begin{array}{l}\text { Environmental } \\
\text { monitoring }\end{array}$ & $\begin{array}{l}\text { Air Particles Concentration, } \\
\text { Ambient Temperature, Altitude, } \\
\text { Atmospheric Pressure, } \mathrm{CO} \text { concentration, } \\
\text { Illuminance, Mass, } \mathrm{NO}_{2} \text { concentration, } \\
\mathrm{O}_{3} \text { concentration, Rainfall, } \\
\text { Relative Humidity, Soil Moisture Tension, } \\
\text { Solar Radiation PAR, } \\
\text { Sound Pressure Level, Soil Temperature, } \\
\text { Wind Direction, Wind Speed }\end{array}$ & $\begin{array}{l}\text { Fixed \& Mobile } \\
\text { Sensors }\end{array}$ & $\begin{array}{l}1000+\text { (fixed) \& } 150 \\
\text { (deployed on public } \\
\text { vehicles) }\end{array}$ \\
\hline $\begin{array}{l}\text { Traffic } \\
\text { monitoring }\end{array}$ & $\begin{array}{l}\text { Vehicle Speed (Average \& Instantaneous), } \\
\text { Traffic Congestion, Traffic Intensity }\end{array}$ & Fixed sensors & $48+$ \\
\hline Bike stops & Bike presence detectors & Fixed sensors & 16 bike stops \\
\hline Bus tracking & $\begin{array}{l}\text { Location (fleet management) }+ \\
\text { Remaining time for the next bus }\end{array}$ & Mobile sensors & $400+$ \\
\hline Taxi stops & $\begin{array}{l}\text { Location (fleet management system) + } \\
\text { Taxis available in each stop }\end{array}$ & Mobile sensors & $50+$ \\
\hline $\begin{array}{l}\text { Garbage } \\
\text { management }\end{array}$ & $\begin{array}{l}\text { Waste container fill level gauge }+ \\
\text { Trash truck (fleet management) }\end{array}$ & $\begin{array}{l}\text { Fixed sensors } \\
\text { (Waste } \\
\text { containers) }+ \\
\text { Mobile sensors } \\
\text { (tracking) }\end{array}$ & $50+$ \\
\hline Indoor parking & Vehicle presence detectors & Fixed sensors & $\begin{array}{l}12 \text { public parking } \\
\text { facilities (managed } \\
\text { by private } \\
\text { companies) }\end{array}$ \\
\hline Outdoor parking & $\begin{array}{l}\text { Vehicle presence detectors } \\
\text { (buried under the asphalt) }\end{array}$ & $\begin{array}{l}\text { Fixed sensors }+ \\
\text { Information } \\
\text { panels }\end{array}$ & $\begin{array}{l}400+\text { sensors \& } 10 \\
\text { panels to display the } \\
\text { information }\end{array}$ \\
\hline $\begin{array}{l}\text { Parks \& gardens } \\
\text { irrigation }\end{array}$ & $\begin{array}{l}\text { Ambient temperature, Atmospheric } \\
\text { Pressure, Rainfall, Relative Humidity, Soil } \\
\text { Moisture Tension, Solar Radiation PAR, } \\
\text { Wind Direction, Wind Speed }\end{array}$ & Fixed sensors & 48 IoT sensors nodes \\
\hline $\begin{array}{l}\text { Presence \& } \\
\text { luminosity }\end{array}$ & $\begin{array}{l}\text { Pedestrian presence detector, Luminosity } \\
\text { Sensors }\end{array}$ & Fixed sensors & 10 \\
\hline NFC \& QR tags & $\begin{array}{l}\text { General information (e.g. transportation, } \\
\text { cultural elements and shops) }\end{array}$ & NFC \& QR Tags & $\begin{array}{l}2000+\text { tags deployed } \\
\text { throughout the city }\end{array}$ \\
\hline $\begin{array}{l}\text { Electromagnetic } \\
\text { exposure }\end{array}$ & $\begin{array}{l}\text { Electric Field in the bands of } 900,1800 \text {, } \\
2100 \text { and } 2400 \mathrm{MHz}\end{array}$ & Fixed sensors & 48 sensor nodes \\
\hline $\begin{array}{l}\text { Augmented } \\
\text { Reality }\end{array}$ & $\begin{array}{l}\text { Contextual information (shops, } \\
\text { restaurants, cultural points of interest, etc.) }\end{array}$ & Points of interest & $2000+$ \\
\hline $\begin{array}{l}\text { Participatory } \\
\text { Sensing }\end{array}$ & $\begin{array}{l}\text { Events generated by citizens (Pace Of The } \\
\text { City) }\end{array}$ & Smartphone apps & $\begin{array}{l}20000+\text { apps } \\
\text { installed into } \\
\text { citizens' } \\
\text { smartphones }\end{array}$ \\
\hline
\end{tabular}


semaphores, information panels, etc., in order to behave as forwarding nodes. In order to avoid forwarding and battery powering of IoT nodes, the repeaters are also equipped with sensors, where possible. The communication between repeaters and the standalone IoT nodes uses 802.15.4 protocol. Around 200 gateways make the third layer. Both repeaters and the IoT nodes send all the information to the gateway using 802.15.4. Gateways use mobile cellular network or $\mathrm{WiFi}$ or Ethernet to transfer the received data from sensors to the testbed core.

\section{Data Analysis of Sensor Reading SeQuences}

In this section we present a statistical analysis of the IoT sensors received measurement data, based on the cross-correlation of the data. The Pearson correlation coefficient [25] is used as the correlation measure:

$$
r=\frac{n \sum_{i=1}^{n} x_{i} y_{i}-\sum_{i=1}^{n} x_{i} \sum_{i=1}^{n} y_{i}}{\sqrt{n \sum_{i=1}^{n} x_{i}^{2}-\left(\sum_{i=1}^{n} x_{i}\right)^{2}} \sqrt{n \sum_{i=1}^{n} y_{i}^{2}-\left(\sum_{i=1}^{n} y_{i}\right)^{2}}}
$$

where $x_{i}$ and $y_{i}$ are $i$-th members of the respective datasets $\mathbf{X}$ and $\mathbf{Y}$, whose correlation coefficient is needed, and $n$ is the number of samples in each dataset.

As an example of the sensors output, Fig. 3 shows temperature measurements of one sensor over time of 37 days. It can be seen that there are some periods of time with missing measurement data. In the following text we will show that the absence of the measurements is a consequence of the ZigBee packet loss due to the interference at the $2.4 \mathrm{GHz}$ frequency band. Since the testbed has deployed sensors for the electrical field (EF) measurements at $2.4 \mathrm{GHz}$, these sensors might be used for the interfering signals detection. Namely, the WiFi signal has much higher power level than the ZigBee signal. Therefore, a high correlation of the $2.4 \mathrm{GHz} E \mathrm{EF}$ measurements with the presence/absence of some other sensor measurements would prove that 1) WiFi interference is the cause of the missing data, 2) EF sensor might be used for the interference detection/prediction. There is a total of $15 \mathrm{EF}$ sensors in the testbed. Thus, we divided the whole network into 15 groups of sensors, and each group consists of the EF sensor and other sensors with up to 50 meters away from the EF sensor. According to (1), Fig. 4 shows the Pearsons correlation coefficient between EF levels and the presence/absence of the measurements from other sensors in the group. Presence/absence of the data is mapped into a dataset $\mathbf{X}$, whose members are zeros and ones, where one means that the data is present, and zero shows that the data is missing. EF data is mapped into the dataset $\mathbf{Y}$. 


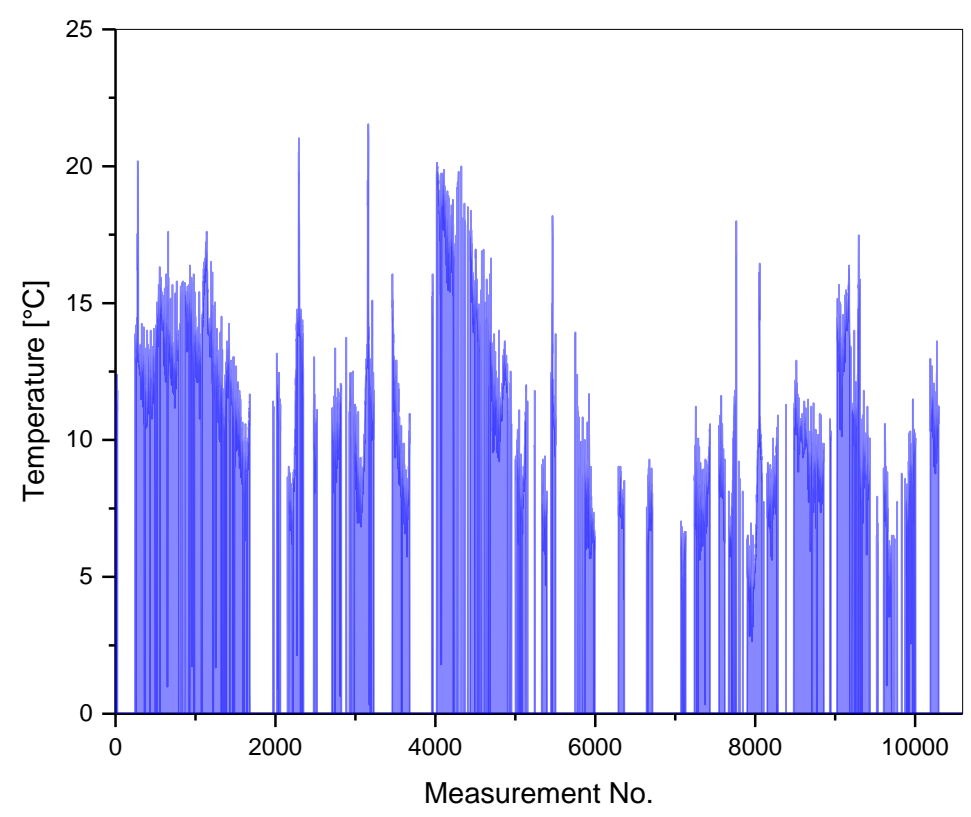

Fig. 3 Temperature sensor measurements over time

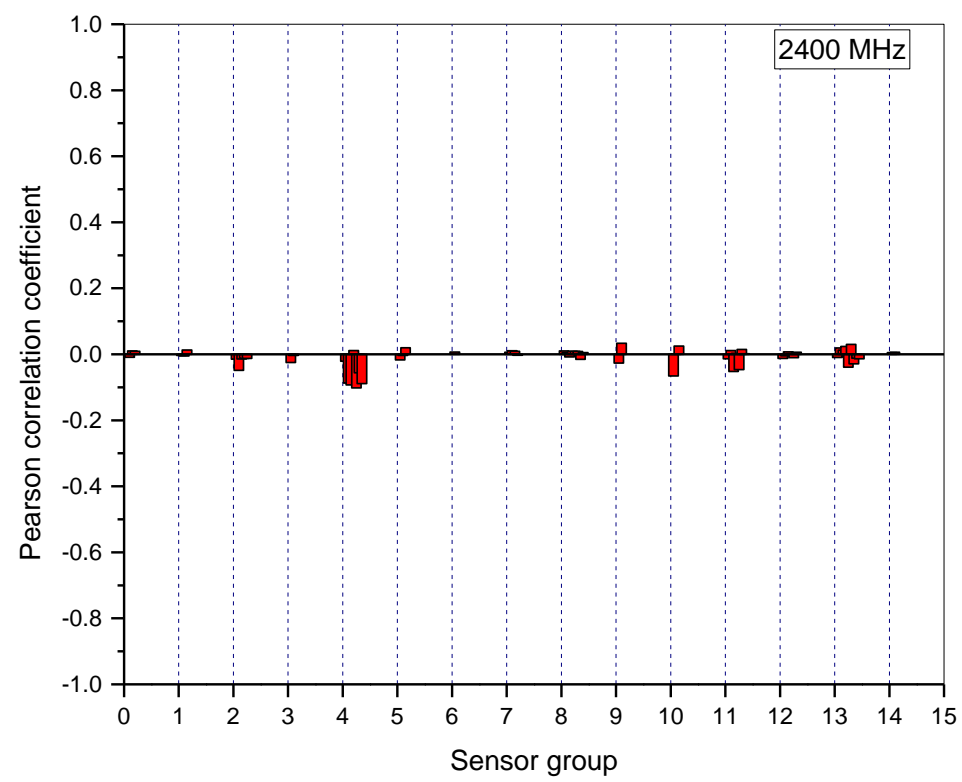

Fig. 4 Pearson correlation coefficient between EF and other sensors presence/absence of data 
Fig. 4 indicates that there is no correlation between the EF level and the other measurement data. The reason for this behavior is that EF sensors are also interfered by $\mathrm{WiFi}$, and therefore they cannot measure high EF field levels. In order to prove that the EF sensor is interfered, we will analyze the correlation between presence/absence of the measurements for different EF sensors at one location. Namely, besides the $2400 \mathrm{MHz} \mathrm{EF}$ sensor, there are 900, 1800 and $2100 \mathrm{MHz}$ sensors at the same location. It is expected that the interference has similar influence on all these sensors. Therefore, Fig. 5 shows the mutual EF sensors correlation for each of the $15 \mathrm{EF}$ sensors groups. It can be seen that the correlation is very high for each EF sensors group. This confirms the assumption that the absence of the measurements data is a consequence of the interference. In order to further confirm this assumption, Fig. 6 shows the correlation between $2400 \mathrm{MHz}$ EF sensor presence/absence of data with the presence/absence of the data from other sensors in the same group. The results show that, due to the high correlation, the most of the sensors are interfered at the same time. However, there are some sensors with low Pearson correlation coefficient. These sensors are either separated from the interference by an obstacle, or they operate at a different ZigBee channel that is not hit by the interference. It should be noted that the interference influences not the sensors themselves (for example, by saturating the levels, or some other mechanism), but instead the communication of the measured levels to the repeater or gateway.

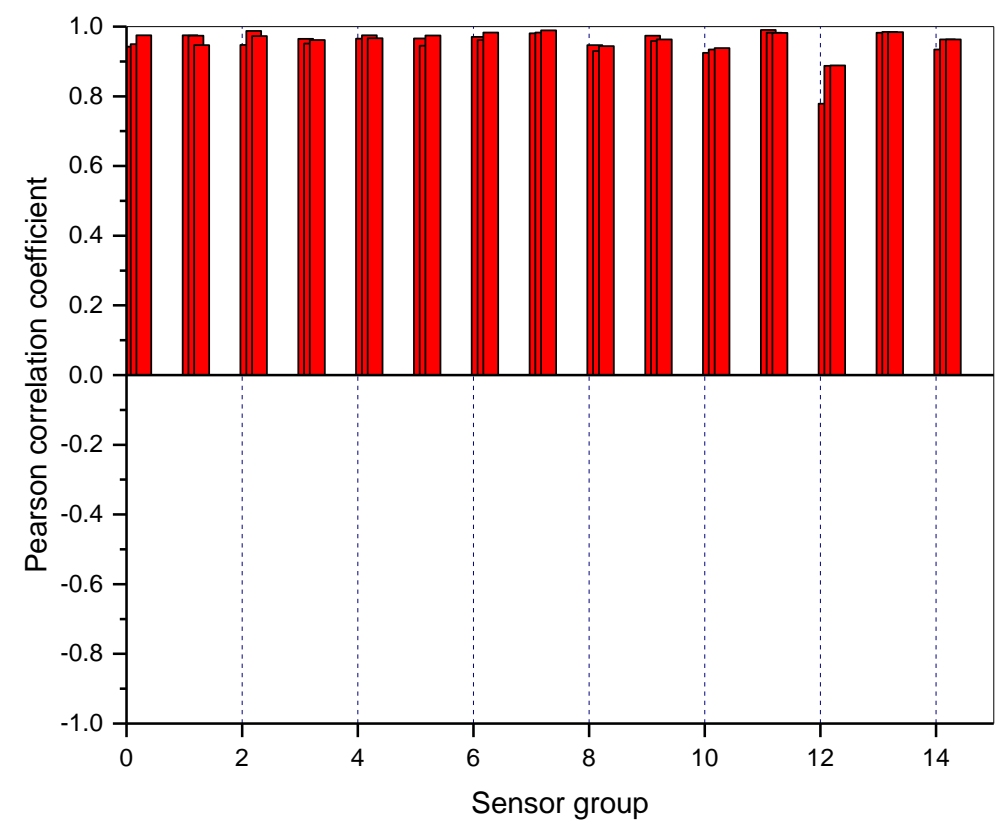

Fig. 5 Mutual EF sensors correlation 


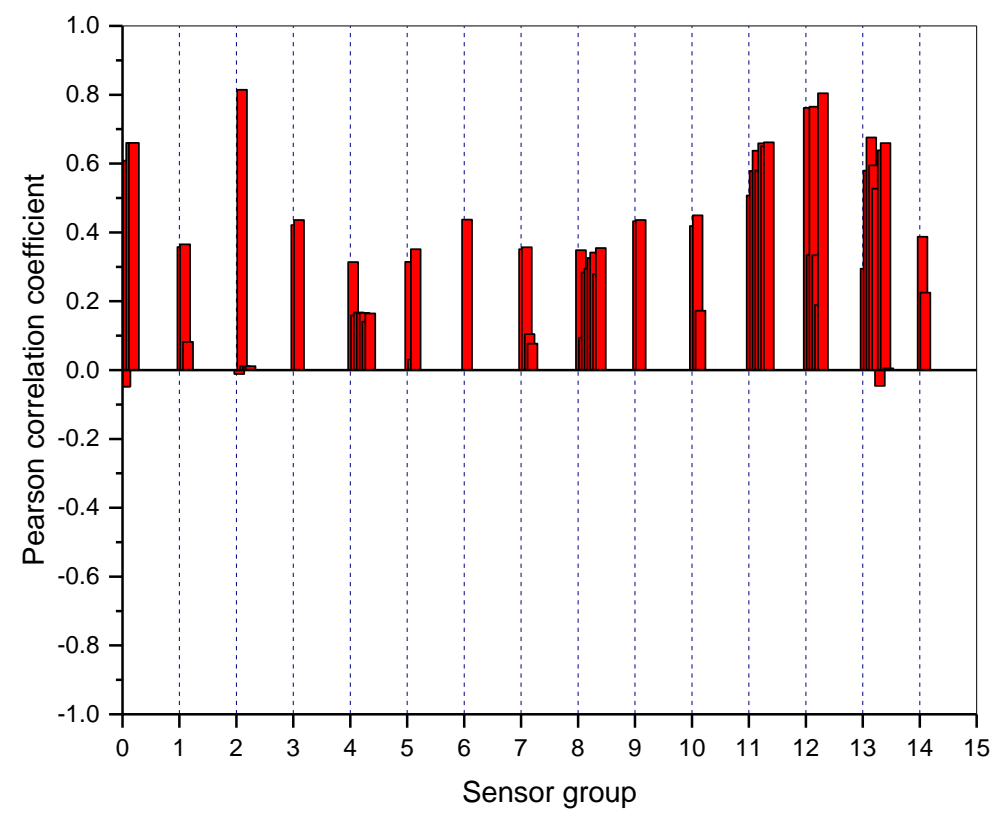

Fig. 6 Correlation between $2400 \mathrm{MHz}$ EF sensor and other sensors in the group presence/absence of the measurement data

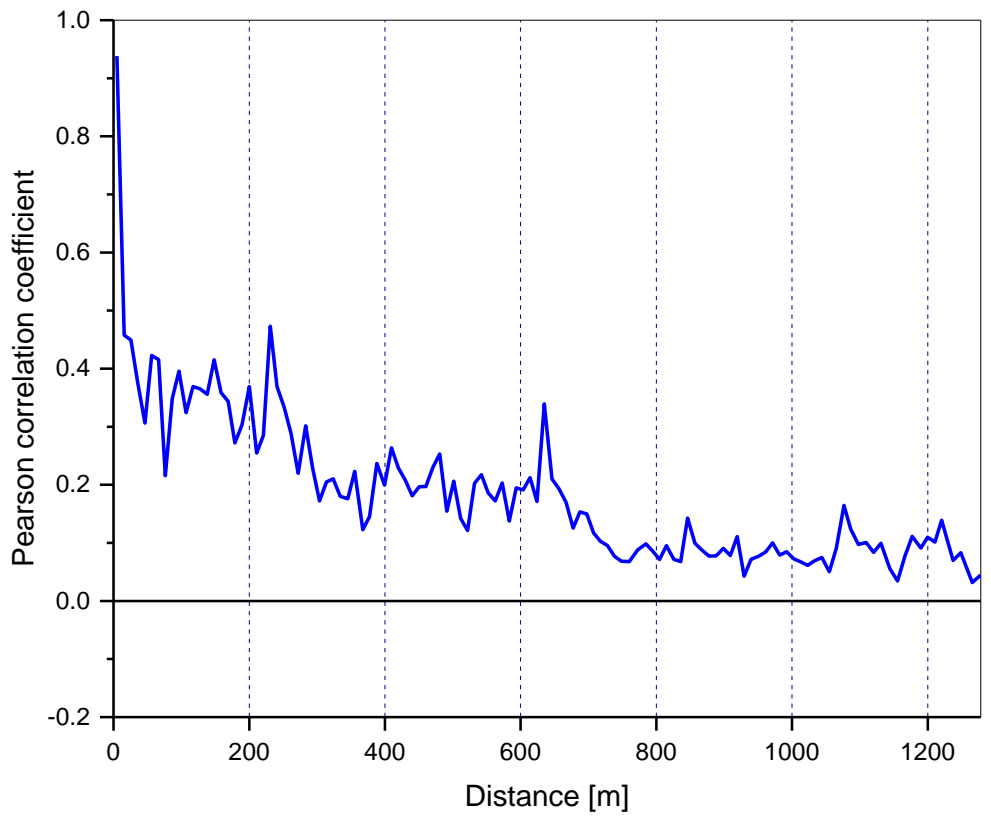

Fig. 7 Pearson correlation coefficient as function of distance between sensors 
Finally, Fig. 7 shows the Pearson correlation coefficient between all pairs of sensors in the network, regardless of their type, as a function of the distance between sensors. The correlation is averaged over the distance of $10 \mathrm{~m}$. It is visible that the correlation decreases over distance, meaning that the sensors close to each other are hit by the same interference, while distant sensors are not.

The presented analysis strongly supports the hypothesis that the absence of the measurement data from the sensors is a consequence of the interference, most likely the WiFi interference. In order to avoid the interference and, consequently, avoid the loss of data, a proactive spectrum coordination [26] might be used. The goal of the proactive spectrum coordination is to prevent the interference between the heterogenous wireless systems by changing the operating channel of a sensor before the interference appears. The coordination process consists of two steps. In the first step, a neural network may be used to analyses measurement data and predict the moment of the interference between $\mathrm{WiFi}$ and ZigBee, based on the presence or absence of the data. On the basis of the predicted interference patterns, the semantic technologies will be used for the reasoning about the needed steps for the coordination.

\section{CONCLUSION}

The problem of coexistence between $\mathrm{WiFi}$ and ZigBee networks within the IoT applications is discussed in this paper. WiFi devices operate at the same $2.4 \mathrm{GHz}$ frequency band as ZigBee, with much higher power, which may lead to the loss of data in the ZigBee network. The analysis is performed on SmartSantander, a massive IoT online testbed, located in Santander, Spain. By analyzing the sensor data, one can notice that there are some missing measurements. It was shown that the data are missing because of the interference. By using neural networks, the time instants of the missing data may be predicted, and the operating frequency of the considered IoT node may be changed in advance, for the sake of the interference avoidance, which will be the subject of the future research.

Acknowledgement: The research leading to these results are performed within the project "SemantiC Coordination for intelligENT sensors (2CENTs)". This project has received funding from the European Union's Horizon 2020 research and innovation programme under grant agreement No 643943. The research was performed on SmartSantander testbed. 


\section{REFERENCES}

[1] M. Weiser, "The Computer for the 21st Century," SIGMOBILE Mob. Comput. Commun. Rev., vol. 3, no. 3, pp. 3-11, 1999, DOI: 10.1145/329124.329126.

[2] J. Pontin, "Bill Joy’s Six Webs," MITTechnology Rev., vol. 29, 2005.

[3] M. R. Palattella et al., "Internet of Things in the 5G Era: Enablers, Architecture, and Business Models," IEEE J. Sel. Areas Commun., vol. 34, no. 3, pp. 510-527, 2016, DOI: 10.1109/JSAC.2016.2525418.

[4] J. Gubbi, R. Buyya, S. Marusic, and M. Palaniswami, "Internet of Things (IoT): A vision, architectural elements, and future directions," Futur. Gener. Comput. Syst., vol. 29, no. 7, pp. 1645-1660, 2013, DOI: https://doi.org/10.1016/j.future.2013.01.010.

[5] A. Al-Fuqaha, M. Guizani, M. Mohammadi, M. Aledhari, and M. Ayyash, "Internet of Things: A Survey on Enabling Technologies, Protocols, and Applications," IEEE Commun. Surv. Tutorials, vol. 17, no. 4, pp. 2347-2376, 2015, DOI: 10.1109/COMST.2015.2444095

[6] T. Kumar and P. B. Mane, "ZigBee topology: A survey," in 2016 International Conference on Control, Instrumentation, Communication and Computational Technologies (ICCICCT), 2016, pp. 164-166.

[7] N. V. R. Kumar, C. Bhuvana, and S. Anushya, "Comparison of ZigBee and Bluetooth wireless technologies-survey," in 2017 International Conference on Information Communication and Embedded Systems (ICICES), 2017, pp. 1-4.

[8] J. r. Lin, T. Talty, and O. K. Tonguz, "On the potential of bluetooth low energy technology for vehicular applications," IEEE Commun. Mag., vol. 53, no. 1, pp. 267-275, 2015, DOI: 10.1109/MCOM.2015.7010544.

[9] C. Gomez, J. Oller, and J. Paradells, "Overview and evaluation of bluetooth low energy: An emerging low-power wireless technology," Sensors, vol. 12, no. 9, pp. 11734-11753, 2012.

[10] "Federated Interoperable Semantic IoT Testbeds and Applications (FIESTA-IoT)." [Online]. Available: http://fiesta-iot.eu/.

[11] L. Sanchez et al., "SmartSantander: IoT Experimentation over a Smart City Testbed," Comput. Netw., vol. 61, pp. 217-238, 2014, DOI: 10.1016/j.bjp.2013.12.020.

[12] G. Anastasi, M. Conti, and M. Di Francesco, "A Comprehensive Analysis of the MAC Unreliability Problem in IEEE 802.15.4 Wireless Sensor Networks," IEEE Trans. Ind. Informatics, vol. 7, no. 1, pp. 52-65, Feb. 2011, DOI: 10.1109/TII.2010.2085440.

[13] P. Huang, L. Xiao, S. Soltani, M. W. Mutka, and N. Xi, "The Evolution of MAC Protocols in Wireless Sensor Networks: A Survey,” IEEE Commun. Surv. Tutorials, vol. 15, no. 1, pp. 101-120, 2013, DOI: 10.1109/SURV.2012.040412.00105

[14] Crossbow Inc., "Avoiding RF Interference Between WiFi and Zigbee." [Online]. Available: https://www.mobiusconsulting.com/papers/ZigBeeandWiFiInterference.pdf.

[15] S. Pollin, I. Tan, B. Hodge, C. Chun, and A. Bahai, "Harmful Coexistence Between 802.15.4 and 802.11: A Measurement-based Study," in 2008 3rd International Conference on Cognitive Radio Oriented Wireless Networks and Communications (CrownCom 2008), 2008, pp. 1-6.

[16] J. Huang, G. Xing, G. Zhou, and R. Zhou, "Beyond co-existence: Exploiting WiFi white space for Zigbee performance assurance," in The 18th IEEE International Conference on Network Protocols, 2010, pp. 305-314.

[17] C.-J. M. Liang, N. B. Priyantha, J. Liu, and A. Terzis, "Surviving Wi-fi Interference in Low Power ZigBee Networks," in Proceedings of the 8th ACM Conference on Embedded Networked Sensor Systems, 2010, pp. 309-322.

[18] X. Zhang and K. G. Shin, "Cooperative Carrier Signaling: Harmonizing Coexisting WPAN and WLAN Devices,” IEEE/ACM Trans. Netw., vol. 21, no. 2, pp. 426-439, Apr. 2013, DOI: 10.1109/TNET. 2012.2200499.

[19] M. S. Kang, J. W. Chong, H. Hyun, S. M. Kim, B. H. Jung, and D. K. Sung, "Adaptive InterferenceAware Multi-Channel Clustering Algorithm in a ZigBee Network in the Presence of WLAN Interference," in 2007 2nd International Symposium on Wireless Pervasive Computing, 2007.

[20] S. Pollin et al., "Distributed cognitive coexistence of 802.15.4 with 802.11," in 2006 1st International Conference on Cognitive Radio Oriented Wireless Networks and Communications, 2006, pp. 1-5.

[21] R. Musaloiu-E. and A. Terzis, "Minimising the Effect of WiFi Interference in 802.15.4 Wireless Sensor Networks,” Int. J. Sen. Netw., vol. 3, no. 1, pp. 43-54, 2008, DOI: 10.1504/IJSNET.2008.016461.

[22] L. Tytgat, O. Yaron, S. Pollin, I. Moerman, and P. Demeester, "Analysis and Experimental Verification of Frequency-Based Interference Avoidance Mechanisms in IEEE 802.15.4," IEEE/ACM Trans. Netw., vol. 23, no. 2, pp. 369-382, Apr. 2015, DOI: 10.1109/TNET.2014.2300114. 
[23] J. W. Chong, C. H. Cho, H. Y. Hwang, and D. K. Sung, “An Adaptive WLAN Interference Mitigation Scheme for ZigBee Sensor Networks,” Int. J. Distrib. Sen. Netw., vol. 2015, p. 159:159--159:159, 2015, DOI: $10.1155 / 2015 / 851289$.

[24] S. Nishikori, K. Kinoshita, Y. Tanigawa, H. Tode, and T. Watanabe, "A cooperative channel control method of ZigBee and WiFi for IoT services," in 2017 14th IEEE Annual Consumer Communications Networking Conference (CCNC), 2017, pp. 1-6.

[25] A. K. Sharma, Text Book Of Correlations And Regression. New Delhi: Discovery Publishing House, 2005.

[26] D. Raychaudhuri, X. Jing, I. Seskar, K. Le, and J. B. Evans, "Cognitive radio technology: From distributed spectrum coordination to adaptive network collaboration," Pervasive and Mobile Computing, vol. 4, no. 3. pp. 278-302, 2008. 